\title{
Adherence to the 'Atrial Fibrillation Better Care' Pathway in Patients with Atrial Fibrillation: Impact on Clinical Outcomes-A Systematic Review and Meta-Analysis of 285,000 Patients
}

\author{
Giulio Francesco Romiti ${ }^{1,2}$ Daniele Pastori ${ }^{3}$ José Miguel Rivera-Caravaca ${ }^{1,4}$ Wern Yew Ding ${ }^{1}$ \\ Ying Xuan Gue ${ }^{1}$ Danilo Menichelli ${ }^{3}$ Jakub Gumprecht ${ }^{1,5}$ Monika Kozieł ${ }^{1,5}$ Pil-Sung Yang ${ }^{6}$ \\ Yutao Guo ${ }^{7(1)}$ Gregory Y.H. Lip ${ }^{1,8, *(1)}$ Marco Proietti ${ }^{1,9,10, *(1)}$
}

${ }^{1}$ Liverpool Centre for Cardiovascular Science, University of Liverpool and Liverpool Heart and Chest Hospital, Liverpool, United Kingdom

${ }^{2}$ Department of Translational and Precision Medicine, Sapienza University of Rome, Rome, Italy

${ }^{3}$ Emergency Medicine Unit, Department of Clinical, Internal, Anesthesiologic and Cardiovascular Sciences, Sapienza University of Rome, Rome, Italy

${ }^{4}$ Department of Cardiology, Hospital Clínico Universitario Virgen de la Arrixaca, Instituto Murciano de Investigación Biosanitaria (IMIBArrixaca), University of Murcia, CIBERCV, Murcia, Spain

5 1st Department of Cardiology and Angiology, Silesian Centre for Heart Diseases, Zabrze, Poland

Thromb Haemost 2022;122:406-414.

Address for correspondence Marco Proietti, MD, PhD, FESC, FEHRA, Geriatric Unit, IRCCS Istituti Clinici Scientifici Maugeri, Via Camaldoli 64, 20138 Milan, Italy (e-mail: marco.proietti@unimi.it).

\footnotetext{
${ }^{6}$ Department of Cardiology, CHA Bundang Medical Center, CHA University, Seongnam, Republic of Korea

7 Department of Cardiology, Medical School of Chinese PLA, Chinese PLA General Hospital, Beijing, China

${ }^{8}$ Department of Clinical Medicine, Aalborg University, Aalborg, Denmark

${ }^{9}$ Geriatric Unit, IRCCS Istituti Clinici Scientifici Maugeri, Milan, Italy

10 Department of Clinical Sciences and Community Health, University of Milan, Milan, Italy
}

\section{Keywords}

- atrial fibrillation

- integrated care

- ABC pathway

- outcomes
Objective The 'Atrial fibrillation Better Care' (ABC) pathway has been recently proposed as a holistic approach for the comprehensive management of patients with atrial fibrillation (AF). We performed a systematic review of current evidence for the use of the $A B C$ pathway on clinical outcomes.

Methods and Results We performed a systematic review and meta-analysis according to PRISMA (Preferred Reporting Items for Systematic Reviews and Meta-Analyses) guidelines. PubMed and EMBASE were searched for studies reporting the prevalence of ABC-pathway-adherent management in AF patients, and its impact on clinical outcomes (all-cause death, cardiovascular death, stroke, and major bleeding). Metaanalysis of odds ratio (OR) was performed with random-effects models; subgroup analysis and meta-regression were performed to account for heterogeneity. Among the eight studies included, we found a pooled prevalence of $A B C$-adherent management of $21 \%$ (95\% confidence interval, $\mathrm{Cl}: 13-34 \%$ ), with a high grade of heterogeneity, explained by the increasing adherence to each $A B C$ criterion. Patients treated according to the $A B C$ pathway showed a lower risk of all-cause death (OR: $0.42 ; 95 \% \mathrm{Cl}$ : $0.31-0.56$ ), cardiovascular death (OR: $0.37 ; 95 \% \mathrm{Cl}: 0.23-0.58)$, stroke (OR: $0.55 ; 95 \%$ $\mathrm{Cl}$ : $0.37-0.82$ ) and major bleeding (OR: $0.69 ; 95 \% \mathrm{Cl}$ : $0.51-0.94)$, with moderate

* Joint senior authors.

received

April 26, 2021

accepted

May 18, 2021

published online

May 21, 2021 (c) 2021. Thieme. All rights reserved. Georg Thieme Verlag KG,

Rüdigerstraße 14,

70469 Stuttgart, Germany
DOI https://doi.org/ 10.1055/a-1515-9630. ISSN 0340-6245. 
heterogeneity. Prevalence of comorbidities was moderators of heterogeneity for allcause and cardiovascular death, while longer follow-up was associated with increased effectiveness for all outcomes.

Conclusion Adherence to the $A B C$ pathway was suboptimal, being adopted in one in every five patients. Adherence to the $A B C$ pathway was associated with a reduction in the risk of major adverse outcomes.

\section{Introduction}

In the last 10 years, great advancements have been made in the treatment of patients with atrial fibrillation (AF), in particular regarding stroke prevention by an increasing use of oral anticoagulant (OAC) drugs. ${ }^{1}$ As a consequence, rates of stroke and thromboembolic events have decreased markedly, being very low in more contemporary cohorts. $^{2}$

Despite the improvements in reducing thromboembolic events, epidemiological data suggest that there were no significant temporal changes to the overall age-adjusted risk of death associated to the presence of $\mathrm{AF}^{3}$ being particularly related to an increase in the risks of hospitalisation and non-cardiovascular (non-CV) death, ${ }^{4}$ also re-emphasising the close relationship between comorbidity, multimorbidity and $\mathrm{AF}^{5,6}$

In order to address the burden of adverse clinical outcomes beyond thromboembolism, implementation of a more comprehensive and integrated approach to AF management has been advociated. ${ }^{7-9}$ To streamline the implementation of such a holistic care approach for AF patients, the 'Atrial fibrillation Better Care' (ABC) pathway has been proposed. The ABC pathway stands on three main pillars: 'A': Avoid stroke (with Anticoagulants); 'B': Better symptom management; ' $C$ ': Cardiovascular and Comorbidity management. ${ }^{9}$ The $A B C$ pathway is now recommended in several clinical guidelines, including the recent European Society of Cardiology (ESC) AF management guidelines. ${ }^{10-12}$

The objective of this article is to present a systematic review of the current evidence for the use of the $A B C$ pathway on clinical outcomes. We aimed, firstly, to establish the overall prevalence of adherence to the $A B C$ criteria in the retrospective analyses available, and secondly, to perform a meta-analysis of $\mathrm{ABC}$ pathway compliance on clinical outcomes.

\section{Methods}

This systematic review has been performed according to the Preferred Reporting Items for Systematic Reviews and MetaAnalyses (PRISMA) guidelines and recommendations (http:// www.prisma-statement.org/). The protocol was registered into the International Prospective Register of Systematic Reviews (PROSPERO; N. CRD42020218088). The data underlying this article are available in the article and in its online Supplementary Material (available in the online version).

\section{Search Strategy}

A systematic and comprehensive literature search was performed on PubMed and EMBASE databases, from inception to December 8, 2020. The search strategy included a combination of key relevant terms related to the research question, including 'ABC Pathway' and 'Atrial Fibrillation Better Care'. The full search strategy is reported in the Supplementary Material (- Supplementary Table S1, available in the online version).

All details regarding study selection, inclusion and exclusion criteria, data extraction, quality assessment and outcomes are reported in the Supplementary Methods (-Supplementary Material, available in the online version).

\section{Statistical Analysis}

Prevalence of ABC-pathway-adherent management was pooled from each of the studies included using a random intercept logistic regression model ${ }^{13}$ with the 'metaprop' function in $\mathrm{R}$.

The number of events and the total number of patients of each group of interest were pooled and compared using a random-effects model. Pooled estimates were reported as odds ratios (ORs) with 95\% confidence intervals (CIs). The inconsistency index $\left(I^{2}\right)$ was calculated to measure heterogeneity. According to pre-specified cut-offs, low heterogeneity was defined as an $I^{2}$ of $<25 \%$, moderate heterogeneity when $I^{2}$ falls between 25 and 75\%, and high heterogeneity when $I^{2}$ was $>75 \%$.

For each outcome, a sensitivity analysis was performed with a 'leave-one-out' approach, in which all studies are removed one at a time to analyse their influence on the pooled estimate and heterogeneity. We also performed several subgroup analyses: (1) for the prevalence of ABCadherent management, according to the geographical location of the original studies; (2) for outcomes (all-cause death, CV death and ischemic stroke), according to pre-specified $\mathrm{CHA}_{2} \mathrm{DS}_{2}$-VASc score groups (i.e., 0-2, 3-5, and 6-9).

To further investigate potential sources of heterogeneity, we performed a meta-regression. Regarding the prevalence of the ABC-pathway-adherent management, we performed a multivariate meta-regression using the adherence to each of the $A B C$ pathway criterion as covariates. For the outcomes, we performed univariate meta-regression with the KnappHartung method ${ }^{14}$ according to the duration of follow-up and thromboembolic risk factors [i.e., age, sex, hypertension, diabetes mellitus, coronary artery disease (CAD), history of stroke, congestive heart failure (CHF)]. 
Publication bias was assessed for studies reporting outcomes according to the adherence to the ABC pathway, with the use of funnel plots, which were visually inspected for asymmetricity. Egger's test was also performed. All the statistical analyses were performed using R (version 4.0.3, The R Foundation, 2020), with the use of 'meta', 'metafor' and 'dmetar' ${ }^{15}$ packages.

\section{Results}

A total of 2,862 results were retrieved from the literature search (761 from PubMed and 2,101 from EMBASE). After the title and abstract screening, 14 full texts were evaluated, and eight studies were included in the final systematic review and meta-analysis ${ }^{16-23}$ (-Table 1 and - Supplementary Fig. S1, available in the online version), with a total of 285,253 AF patients included. Four studies were based in Europe, ${ }^{16-18,20}$ two in Asia, ${ }^{22,23}$ one in North America ${ }^{19}$ and one was multinational. ${ }^{21}$

Among the eight included studies, two were post-hoc subgroup analyses of previously performed randomised controlled trials (RCTs), ${ }^{19,21}$ four were based on observational registries, ${ }^{16-18,20}$ while the last one was derived from a nationwide claims registry. ${ }^{22}$ One study did not include a follow-up phase. ${ }^{17}$ The only RCT, the 'mobile Atrial Fibrillation Application II' (mAFA-II), a cluster-randomised study conducted in China, implemented the $\mathrm{ABC}$ pathway through a mobile phone application. Six out of eight studies ${ }^{17-21,23}$ enrolled both out- and in-patients, while the remaining two only considered hospitalised patients. ${ }^{16,22}$

\section{Prevalence of ABC-Pathway-Adherent Care}

Among the seven observational studies included in the systematic review, we found a pooled prevalence of a clinical management adherent to the $\mathrm{ABC}$ pathway criteria equal to $21 \%$ (95\% CI: $13-34 \%)$, with a high heterogeneity $\left(I^{2}=100 \%\right.$; - Fig. 1$)$. In order to evaluate the factors accounting for such a high degree of heterogeneity, we performed a multivariate meta-regression analysis (-Supplementary Table 52, available in the online version). Among the factors included in the multivariate analysis, adherence to the ' $A$ ', ' $B$ ' and ' $C$ ' components of the $\mathrm{ABC}$ pathway were found to be directly associated with the prevalence of ABC-pathwayadherent clinical management (- Supplementary Table S2, available in the online version). The final model was able to explain most of the heterogeneity observed $\left(R^{2}=98.9 \%\right.$, $p=0.004)$.

\section{Impact of ABC-Adherent Care on Outcomes}

We performed a meta-analysis on the impact of the $A B C$ pathway on major clinical outcomes (-Fig. 2). Overall, the use of ABC-pathway-adherent care was associated with a significant reduction of all-cause death compared with nonadherence (OR: 0.42; 95\% CI: 0.31-0.56), with a high between-studies heterogeneity $\left(I^{2}=88 \%\right.$; - Fig. 2A). The risk of $\mathrm{CV}$ death was significantly lower in patients treated adherent to the $\mathrm{ABC}$ pathway (OR: $0.37 ; 95 \% \mathrm{CI}: 0.23-0.58$ ) with a high degree of heterogeneity $\left(I^{2}=89 \%\right.$; - Fig. 2B).

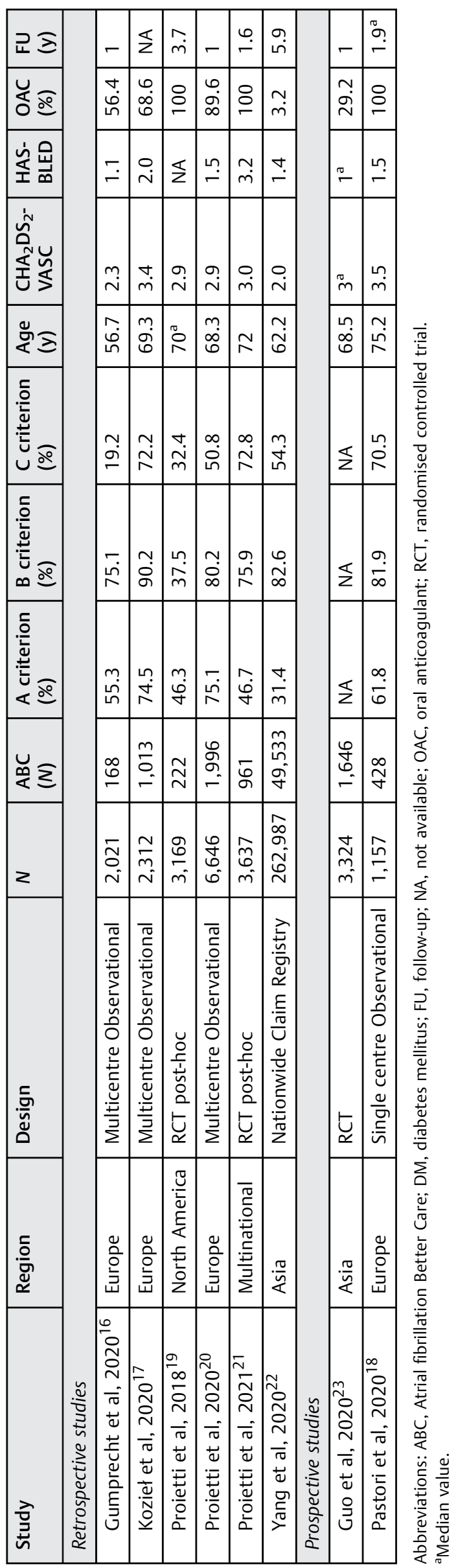




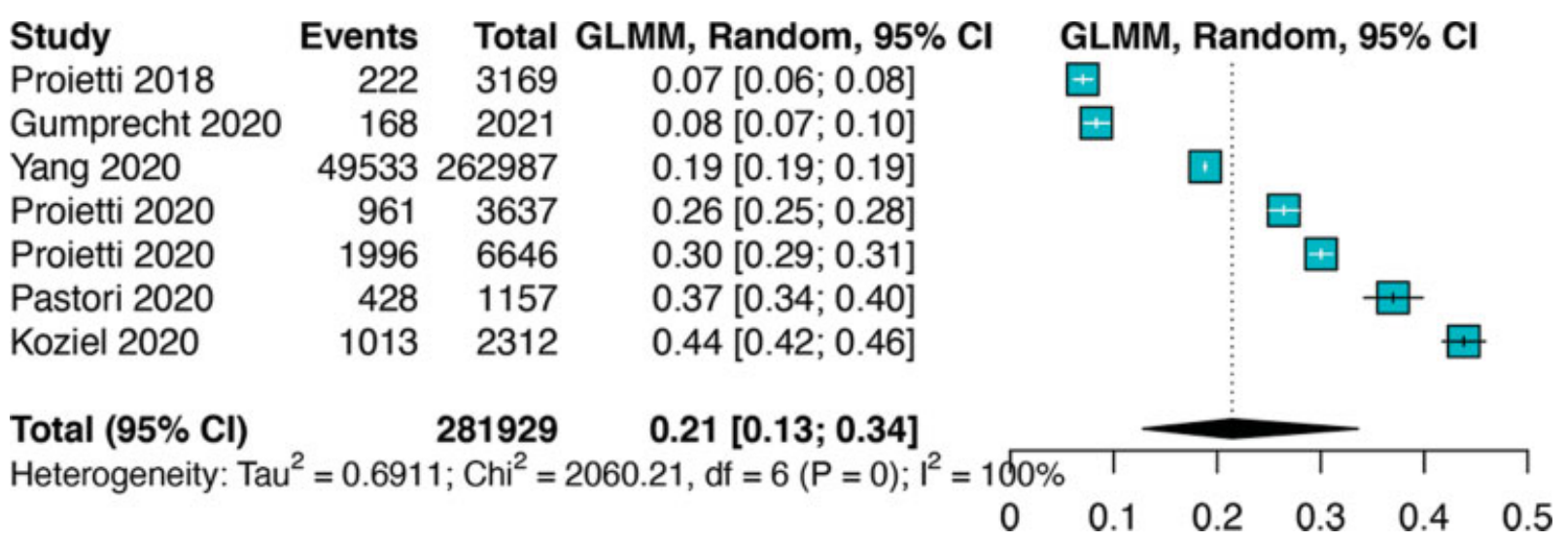

Fig. 1 Pooled prevalence of $A B C$ adherent management. ABC, Atrial fibrillation Better Care; $\mathrm{Cl}$, confidence interval; GLMM, generalised linear mixed model.

The risk of stroke (OR: 0.55; 95\% CI: 0.37-0.82; - Fig. 2C) and major bleeding (OR: 0.69; 95\% CI: 0.51-0.94; - Fig. 2D) were significantly lower in those patients who were treated adherent to the $A B C$ pathway management, with an overall moderate degree of heterogeneity.

\section{Subgroup Analyses}

In order to evaluate the high degree of heterogeneity for the all-cause death outcome, we performed a subgroup analysis in relation to the geographic location of the patients in each study (-Fig. 3) (for one study, which was multinational, we analysed the results according to the regions included). While we did not find a significant difference between European, Asian and North American patients, this analysis found that the geographic location accounted for most of the heterogeneity in the main model, with a $40 \%$ residual heterogeneity.

\section{Meta-regression Analysis}

We performed a univariate meta-regression analysis to examine the relationship between the clinical variables and the association of adherence to the $A B C$ pathway with the risk of all-cause death and CV death. In these analyses (- Supplementary Tables $\mathbf{S 3}$ and S4, available in the online version), we found a direct association between the length of follow-up and an increase in effectiveness, while conversely an increasing prevalence of diabetes mellitus, $\mathrm{CAD}, \mathrm{CHF}$ and stroke was associated with a reduction in effectiveness of the $A B C$ pathway for both all-cause death and CV death occurrence, all accounting for most of the heterogeneity for the two outcomes (-Supplementary Tables $\mathbf{5 3}$ and 54, available in the online version). Furthermore, the meta-regression analysis for stroke (-Supplementary Table S5, available in the online version) and major bleeding (-Supplementary Table S6, available in the online version) found a direct association between the length of follow-up and an increase in effectiveness for both these outcomes, accounting for a significant proportion of heterogeneity (- Supplementary Tables S5 and S6, available in the online version).

\section{Sensitivity Analysis}

The sensitivity analysis for the four outcomes according to the 'leave-one-out' approach did not show any significant differences for each study included and any outcome (-Supplementary Figs. S2-S5, available in the online version). In the $\mathrm{CHA}_{2} \mathrm{DS}_{2}$-VASc-stratified analysis (-Fig. 4), we found that for all-cause death, increasing $\mathrm{CHA}_{2} \mathrm{DS}_{2}$-VASc strata was associated with a progressively greater reduction of risk amongst patients adherent to the $\mathrm{ABC}$ pathway, being greatest at the highest $\mathrm{CHA}_{2} \mathrm{DS}_{2}$-VASc strata (OR: 0.30; $95 \%$ CI: 0.17-0.54 for $\mathrm{CHA}_{2} \mathrm{DS}_{2}$-VASc 6-9) (-Fig. 4A). No difference in $A B C$ pathway effectiveness was found across $\mathrm{CHA}_{2} \mathrm{DS}_{2}$-VASc strata for $\mathrm{CV}$ death and stroke occurrence (-Fig. 4B, C).

\section{Bias Assessment}

The risk of bias assessment (- Supplementary Tables S7 and s8, available in the online version) showed an overall high quality of studies, with the exception of Yang et al, ${ }^{22}$ which was found at high risk of bias for both prevalence and outcomes analysis.

Significant publication bias was found for all-cause death (Egger's test, $p=0.021$ ) and stroke (Egger's test, $p=0.008$, -Supplementary Table 59, available in the online version). Visual inspection of the funnel plots (-Supplementary Fig. S6A, C, available in the online version) revealed that, in both cases, asymmetricity was caused by a void in the left side of the funnel plot, in which one would expect to find studies with positive results. The addition of these potential studies may lead to lower pooled ORs for both all-cause death and stroke.

\section{Discussion}

Firstly, in this systematic review and meta-analysis, clinical management adherent to the $\mathrm{ABC}$ pathway was suboptimal, being adopted in one of every five AF patients. Secondly, meta-analysis regarding clinical events showed that adherence to the $A B C$ pathway was associated with a significant reduction in the risk of major adverse outcomes (-Fig. 5). 


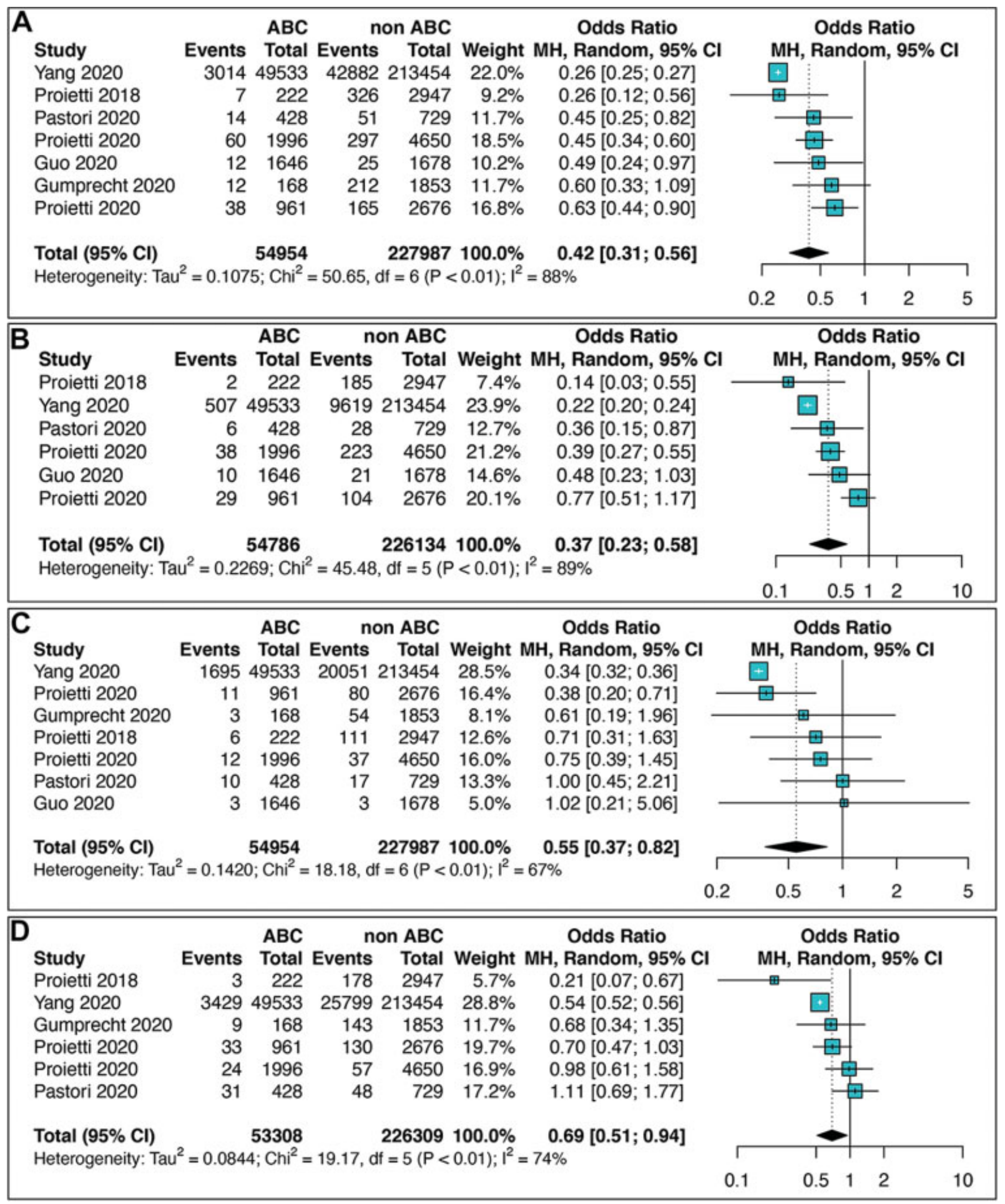

Fig. 2 Impact of $A B C$ adherent management on outcomes. (A) All-cause death; (B) cardiovascular death; (C) stroke; (D) major bleeding. ABC, Atrial fibrillation Better Care; $\mathrm{Cl}$, confidence interval; $\mathrm{MH}$, Mantel-Haenszel.

Thirdly, adherence to the ABC pathway was largely driven by the implementation of adequate antithrombotic therapy, adequate symptom control and by optimal control of $\mathrm{CV}$ risk factors and comorbidities. Lastly, the meta-regression analyses regarding outcomes showed that the increasing clinical complexity directly affects the effectiveness of an integrated management strategy, while a longer follow-up was associated to a greater reduction in risk.
Epidemiologically, the worldwide impact of AF has increased in the last 40 years. Despite a significant reduction in age-standardised prevalence and incidence, the absolute number of AF patients has almost doubled, being significantly increased in countries with middle and low sociodemographic levels. ${ }^{3}$ Additionally, observational studies have shown that the clinical risk profile of AF patients has worsened over time, due to an increase in the prevalence of 


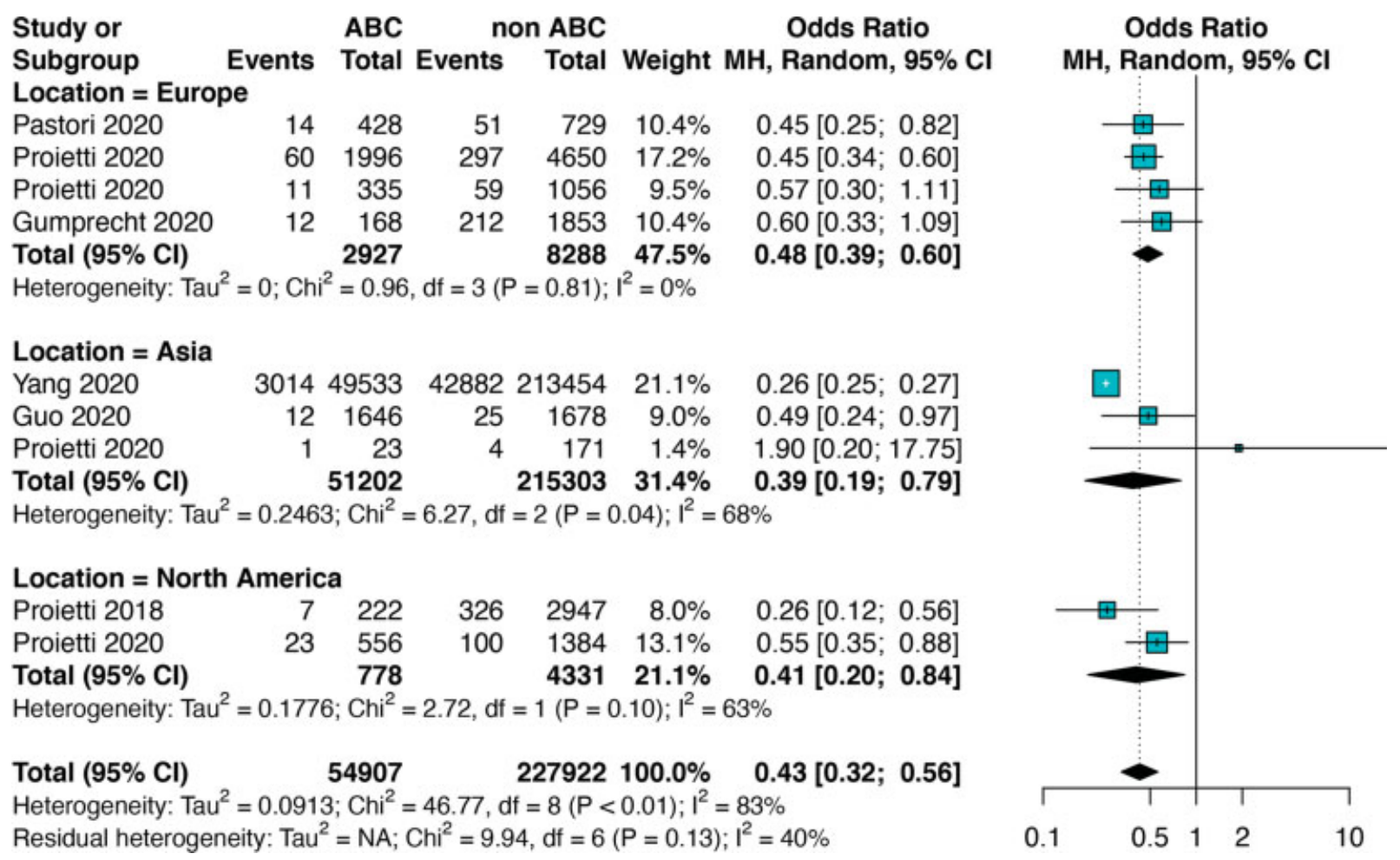

Fig. 3 Impact of $A B C$ adherent management on all-cause death according to regions. ABC, Atrial fibrillation Better Care; $\mathrm{Cl}$, confidence interval; $\mathrm{MH}$, Mantel-Haenszel.
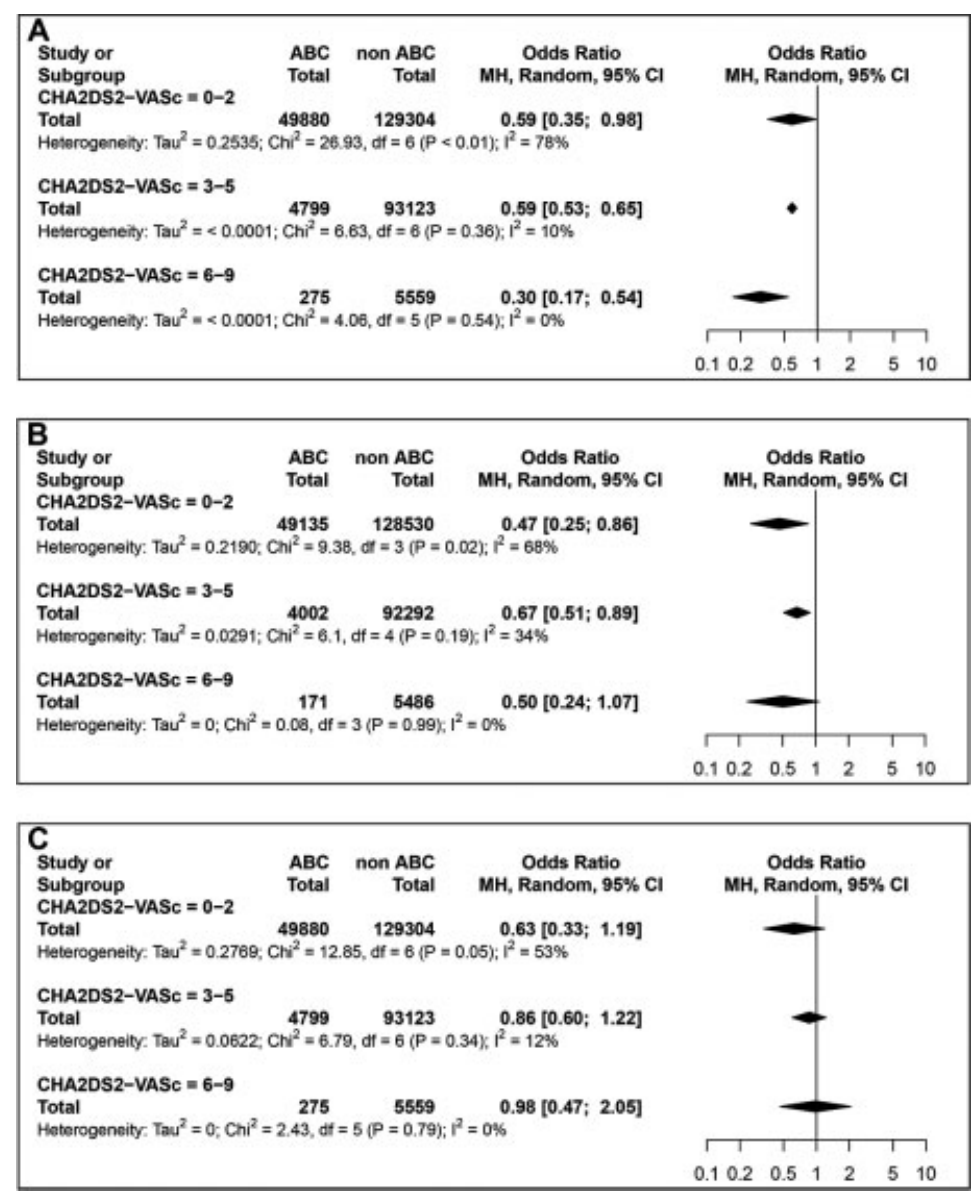

Fig. 4 Impact of $A B C$ according to $\mathrm{CHA}_{2} \mathrm{DS}_{2}$-VASc strata on outcome. (A) All-cause death; (B) cardiovascular death; (C) stroke. ABC, Atrial fibrillation Better Care; $\mathrm{Cl}$, confidence interval; $\mathrm{MH}$, Mantel-Haenszel. 

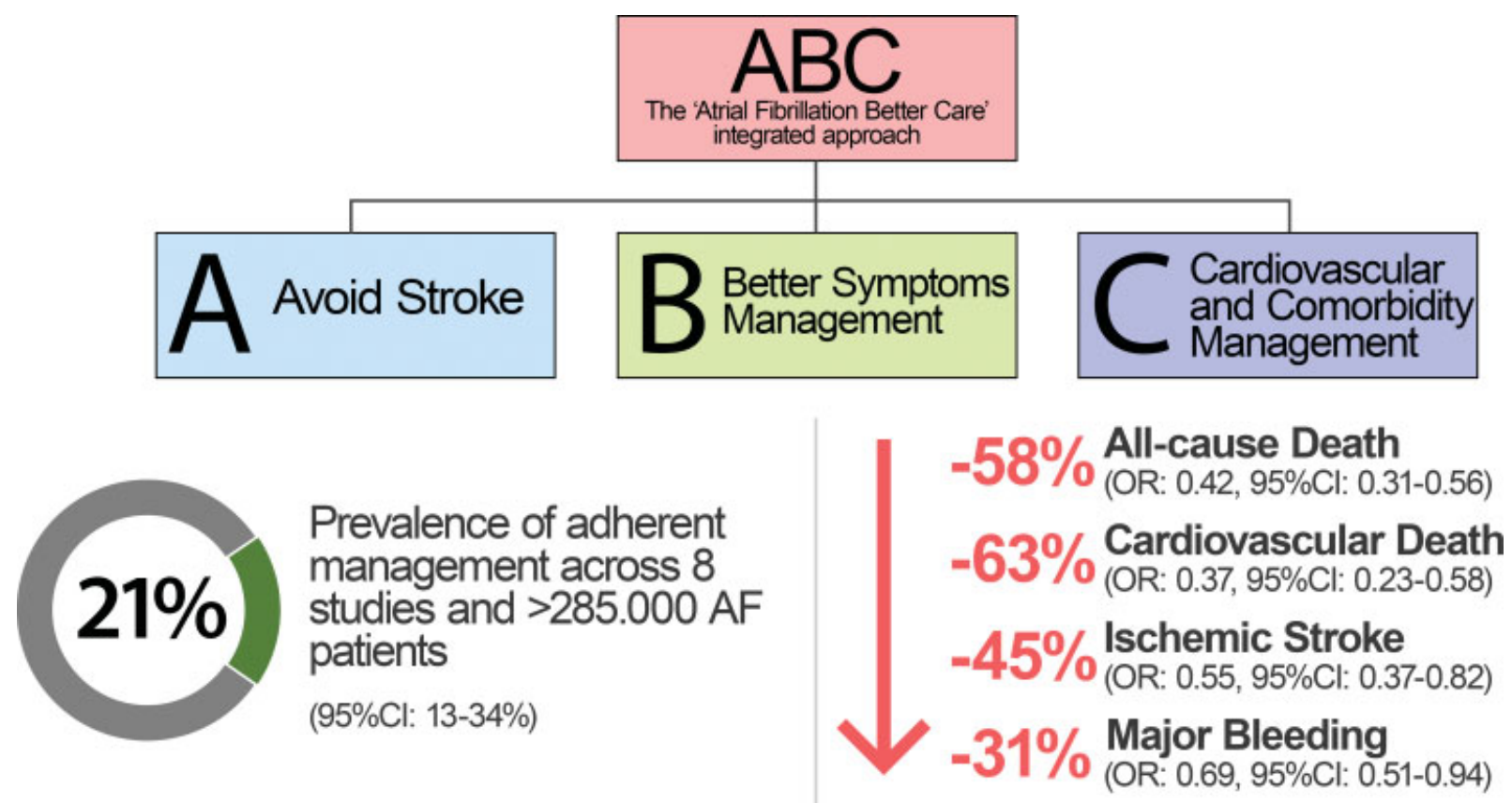

Fig. 5 Graphical synopsis of the main study results. $\mathrm{Cl}$, confidence intervals; OR, odds ratio.

comorbidities. $^{24}$ This public health burden of AF has a major impact on mortality, where the total number of attributable deaths has more than doubled, almost reaching 300,000 in 2017, especially in middle and low socio-demographic countries. $^{3}$ Furthermore, an increasing effect on health-careassociated costs has been found. ${ }^{25,26}$

Given these concerns, a more holistic approach to AF management was needed. In 2018, the 6th AFNET/EHRA Consensus Conference defined integrated care as 'a coordinated patient-centred approach by interdisciplinary specialists to improve AF outcomes', by improving all the specific domains related to AF management. ${ }^{7,27}$ A model of care was proposed in which any AF patient should ideally be managed by the AF Heart Team (specifically addressing the specific electrophysiology/cardiology issues) and the Integrated Care AF Clinic, where several specialists could provide the specific expertise to handle any aspect of the patient's care. $7,8,28$

In 2017, the $A B C$ pathway was proposed to streamline and simplify the implementation of integrated management in AF patients. ${ }^{9}$ This simple model focuses on three main components, which are all essential to reduce the risk of major adverse outcomes in AF. The 'A' criterion (Avoid stroke) refers to the management of thromboembolic and bleeding risks by appropriate prescription and use of OAC drugs; the 'B' criterion (Better symptom management) aims to reduce and control symptom burden and patient-centred, symptom-directed decisions on rate or rhythm control therapy; the ' $\mathrm{C}$ ' criterion (Cardiovascular and Comorbidity risk optimisation) refers to the optimised management of any concomitant comorbidity or CV risk factor.

Our systematic review demonstrates that a significant amount of evidence has already been produced regarding the potential role of the $A B C$ pathway in mitigating the risk of major adverse outcomes. The retrospective analyses showed that the level of adherence to the ABC pathway was low, with just one-fifth of the patients being managed optimally. In particular, our meta-regression results suggest that more efforts are needed to obtain more optimal adherence to all the $\mathrm{ABC}$ pathway components: for example, implementation of adequate antithrombotic therapy and by more optimal control of CV risk factors and comorbidities. However, we cannot exclude that some factors not considered in this analysis may affect the integrated care of AF patients, such as education level, health perception, household income, availability of a public health care system, distance from health care services/hospitals and presence of caregiver for patients with disability. ${ }^{28,29}$ Furthermore, while it is important to underline that a clear heterogeneity exists in the various definitions of 'ABC-pathway-adherent care' across the retrospective studies (as further reported below), the importance of our results stands in the fact that irrespective of how the studies defined components of the $A B C$ pathway, good control of anticoagulation quality, improved control of symptoms burden and the proper management of the most relevant comorbidities require an effort from the treating physician which is 'integrated or holistic care'. This article shows how few patients are clinically managed in this way.

The pivotal role of a holistic approach to AF care is underlined by the results of this meta-analysis, showing that all the major adverse outcomes are significantly and consistently reduced in the ABC-pathway-adherent group of patients. Indeed, ABC-pathway-adherent care was associated with a 40 to $60 \%$ risk reduction for all the outcomes considered. Moreover, the positive results of the only RCT about the $\mathrm{ABC}$ pathway produced strengthen the view that more effort should be put into translating this evidence-based approach into daily clinical practice. Our evidence that a longer observation is associated with a greater reduction in risk for all the outcomes corroborates the evidence for the effectiveness of the intervention, where there is a 'dose-response' effect, 
extending the evidence from the mAFA-II trial secondary analyses. ${ }^{30}$ The negative impact of a higher prevalence of several comorbidities on the effectiveness of ABC-pathwayadherent care emphasises the role of comorbidities and increased clinical complexity in influencing the clinical course. Indeed, an increasing prevalence of diabetes mellitus, CAD, CHF and stroke was associated with a reduction in effectiveness. Such evidence is reinforced by several studies illustrating how an increasing level of multimorbidity is independently associated with an increased risk of outcomes, also determining a differential approach in OAC prescription. ${ }^{5,6}$ In our study, the results of the $\mathrm{CHA}_{2} \mathrm{DS}_{2}$ VASc-stratified analysis showed a greater risk reduction for all-cause death in patients with the highest thromboembolic risk; looking at this evidence and given prior evidence regarding the specific impact of $\mathrm{ABC}$-pathway-adherent care in reducing the risk of outcomes in 'clinically complex' AF patients, for example those with multimorbidity, ${ }^{31}$ we would suggest that such a holistic approach is even more needed in those with the highest risk profiles.

This evidence, together with other data generated by secondary analyses of the studies included in this systematic review, which showed a significant impact of the ABC-pathway-adherent care in reducing the risk of demen$\mathrm{tia}^{32}$ and a significant reduction in health-care-associated costs, ${ }^{33}$ strongly supports the recent changes introduced in the 2020 ESC AF clinical guidelines. ${ }^{12}$ In these guidelines, there is a paradigm shift in approach, placing the patient at the centre of the physicians' action, not the disease itself. With the aim of managing the patient holistically, the application of the $\mathrm{ABC}$ pathway is central to the guideline recommendations.

\section{Limitations}

Our article has some limitations. First, the observational and retrospective nature of most of the included studies inherently limits the generalisability of the results. Even though we performed several meta-regression analyses, unmeasured residual confounders may have influenced our results given that the data were mostly from observational studies. Furthermore, since two of the studies included were originally performed more than 10 years ago, the different clinical practices could have impacted the overall rate of adverse outcomes. Another major limitation which we can recognise is related to an inevitable heterogeneity in the $A B C$ pathway criterion definition, particularly in relation to the ' $\mathrm{B}$ ' criterion, which varied significantly between the studies. Notwithstanding this, it should be taken in mind that in the spirit of the original $A B C$ pathway proposal, the point is related to the best control of the particular criterion in the individual studies, irrespective of the methods used to obtain the control or compliance with uniform targets, and to evaluate the effect on outcomes. Even though there was heterogeneity of assessments used in each study, the evaluation of the $A B C$ criteria aimed to identify patients who were best managed to obtain the best control possible for each criterion. Lastly, for the evaluation of the ' $\mathrm{C}$ ' criterion, most of the studies limited the evaluation to the main $\mathrm{CV}$ risk factors and comorbidities.

\section{Conclusion}

In this systematic review and meta-analysis, clinical management adherent to the $\mathrm{ABC}$ pathway was sub-optimally applied, being adopted in one in every five AF patients. Adherence to the ABC pathway was associated with a significant reduction in the risk of major adverse outcomes, with a significantly reduced risk of all-cause death, CV death, stroke and major bleeding.

\section{What is known about this topic?}

- In atrial fibrillation (AF) patients, multimorbidity and clinical complexity increase the risk of death and hospitalisation.

- In recent years the need for a more comprehensive and holistic approach to AF patients has been recognised.

- The 'Atrial fibrillation Better Care' (ABC) pathway has been proposed to streamline the application of integrated or holistic care in AF patients.

\section{What does this paper add?}

- In this systematic review and meta-analysis, we show that a clinical management adherent to the $\mathrm{ABC}$ pathway was sub-optimally applied in AF patients.

- ABC-pathway-adherent care was associated with a significant reduction of all-cause death, cardiovascular death, stroke and major bleeding risk in AF patients.

- Increased clinical complexity decreases the effectiveness of the $A B C$ pathway in reducing risk of death, while a longer follow-up time maximises the effect of an integrated care approach.

\section{Conflict of Interest}

J.M.R.-C.: Recipient of grants from Sociedad Española de Trombosis y Hemostasia (grant for short international training stays 2020) and the First Contact Initiative Grant 2020 from the European Society of Cardiology Council on Basic Cardiovascular Science. G.Y.H.L.: Consultant and speaker for BMS/Pfizer, Boehringer Ingelheim and Daiichi-Sankyo. No fees were received personally. The other authors declare no significant conflict of interest.

\section{References}

1 Proietti M, Lane DA, Boriani G, Lip GYH. Stroke prevention, evaluation of bleeding risk, and anticoagulant treatment management in atrial fibrillation contemporary international guidelines. Can J Cardiol 2019;35(05):619-633

2 Boriani G, Proietti M, Laroche C, et al; EORP-AF Long-Term General Registry Investigators. Association between antithrombotic treatment and outcomes at 1-year follow-up in patients with atrial fibrillation: the EORP-AF General Long-Term Registry. Europace 2019;21(07):1013-1022

3 Dai $\mathrm{H}$, Zhang Q, Much AA, et al. Global, regional, and national prevalence, incidence, mortality, and risk factors for atrial fibrillation, 1990-2017: results from the Global Burden of Disease Study 2017. Eur Heart J Qual Care Clin Outcomes 2020 (e-pub ahead of print). Doi: 10.1093/ehjqcco/qcaa061 
4 Singh SM, Abdel-Qadir H, Pang A, et al. Population trends in allcause mortality and cause specific-death with incident atrial fibrillation. J Am Heart Assoc 2020;9(19):e016810

5 Proietti M, Marzona I, Vannini T, et al. Long-term relationship between atrial fibrillation, multimorbidity and oral anticoagulant drug use. Mayo Clin Proc 2019;94(12):2427-2436

6 Alexander KP, Brouwer MA, Mulder H, et al; ARISTOTLE Investigators. Outcomes of apixaban versus warfarin in patients with atrial fibrillation and multi-morbidity: Insights from the ARISTOTLE trial. Am Heart J 2019;208:123-131

7 Kotecha D, Breithardt G, Camm AJ, et al. Integrating new approaches to atrial fibrillation management: the 6th AFNET/EHRA Consensus Conference. Europace 2018;20(03): 395-407

8 Kirchhof P. The future of atrial fibrillation management: integrated care and stratified therapy. Lancet 2017;390(10105): 1873-1887

9 Lip GYH. The ABC pathway: an integrated approach to improve AF management. Nat Rev Cardiol 2017;14(11):627-628

10 Lip GYH, Banerjee A, Boriani G, et al. Antithrombotic therapy for atrial fibrillation: CHEST guideline and expert panel report. Chest 2018;154(05):1121-1201

11 Joung B, Lee JM, Lee KH, et al; KHRS Atrial Fibrillation Guideline Working Group. 2018 Korean guideline of atrial fibrillation management. Korean Circ J 2018;48(12):1033-1080

12 Hindricks G, Potpara T, Dagres N, et al; ESC Scientific Document Group. 2020 ESC Guidelines for the diagnosis and management of atrial fibrillation developed in collaboration with the European Association for Cardio-Thoracic Surgery (EACTS): The Task Force for the diagnosis and management of atrial fibrillation of the European Society of Cardiology (ESC) developed with the special contribution of the European Heart Rhythm Association (EHRA) of the ESC. Eur Heart J 2021;42(05):373-498

13 Stijnen T, Hamza TH, Özdemir P. Random effects meta-analysis of event outcome in the framework of the generalized linear mixed model with applications in sparse data. Stat Med 2010;29(29): 3046-3067

14 Knapp G, Hartung J. Improved tests for a random effects metaregression with a single covariate. Stat Med 2003;22(17): 2693-2710

15 Harrer M, Cuijpers P, Furukawa TA, et al. Doing Meta-analysis in R: A Hands-on Guide. Erlangen: Prot Lab; 2019

16 Gumprecht J, Domek M, Proietti M, et al. Compliance of atrial fibrillation treatment with the Atrial fibrillation Better Care (ABC) pathway improves the clinical outcomes in the Middle East population: a report from the Gulf Survey of Atrial Fibrillation Events (SAFE) registry. J Clin Med 2020;9(05):1286

17 Kozieł M, Simovic S, Pavlovic N, et al. Adherence to the ABC (Atrial fibrillation Better Care) pathway in the Balkan region: the BALKAN-AF survey. Pol Arch Intern Med 2020;130(03):187-195

18 Pastori D, Menichelli D, Violi F, et al. The Atrial fibrillation Better Care $(A B C)$ pathway and cardiac complications in atrial fibrillation: a potential sex-based difference. The ATHERO-AF study. Eur J Intern Med 2020;85:80-85

19 Proietti M, Romiti GF, Olshansky B, Lane DA, Lip GYH. Improved outcomes by integrated care of anticoagulated patients with atrial fibrillation using the simple ABC (Atrial Fibrillation Better Care) pathway. Am J Med 2018;131(11):1359.e6-1366.e6

20 Proietti M, Lip GYH, Laroche C, et al; ESC-EORP Atrial Fibrillation General Long-Term Registry Investigators Group. Relation of outcomes to ABC (Atrial Fibrillation Better Care) pathway adherent care in European patients with atrial fibrillation: an analysis from the ESC-EHRA EORP Atrial Fibrillation General Long-Term (AFGen LT) Registry. Europace 2021;23(02):174-183

21 Proietti M, Vitolo M, Lip GYH. Integrated care and outcomes in patients with atrial fibrillation and comorbidities. Eur J Clin Invest 2021;51(06):e13498

22 Yang PS, Sung JH, Jang E, et al. Application of the simple atrial fibrillation better care pathway for integrated care management in frail patients with atrial fibrillation: a nationwide cohort study. J Arrhythm 2020;36(04):668-677

23 Guo Y, Lane DA, Wang L, et al; mAF-App II Trial Investigators. Mobile health technology to improve care for patients with atrial fibrillation. J Am Coll Cardiol 2020;75(13):1523-1534

24 Proietti M, Laroche C, Nieuwlaat R, et al; EORP-AF General Pilot Registry Euro Heart Survey on AF Investigators. Increased burden of comorbidities and risk of cardiovascular death in atrial fibrillation patients in Europe over ten years: a comparison between EORP-AF pilot and EHS-AF registries. Eur J Intern Med 2018; 55:28-34

25 Rahman F, Kwan GF, Benjamin EJ. Global epidemiology of atrial fibrillation. Nat Rev Cardiol 2014;11(11):639-654

26 Burdett P, Lip GYH. Atrial fibrillation in the United Kingdom: predicting costs of an emerging epidemic recognising and forecasting the cost drivers of atrial fibrillation-related costs. Eur Heart J Qual Care Clin Outcomes 2020. Doi: 10.1093/ehjqcco/qcaa093

27 Kirchhof P, Benussi S, Kotecha D, et al; ESC Scientific Document Group. 2016 ESC Guidelines for the management of atrial fibrillation developed in collaboration with EACTS. Eur Heart J 2016;37 (38):2893-2962

28 Rivera-Caravaca JM, Gil-Perez P, Lopez-García C, et al. A nurse-led atrial fibrillation clinic: impact on anticoagulation therapy and clinical outcomes. Int J Clin Pract 2020;74(12):e13634

29 LaRosa AR, Claxton J, O'Neal WT, et al. Association of household income and adverse outcomes in patients with atrial fibrillation. Heart 2020;106(21):1679-1685

30 Guo Y, Guo J, Shi X, et al; mAF-App II Trial investigators. Mobile health technology-supported atrial fibrillation screening and integrated care: a report from the mAFA-II trial Long-term Extension Cohort. Eur J Intern Med 2020;82:105-111

31 Proietti M, Romiti GF, Olshansky B, Lane DA, Lip GYH. Comprehensive management with the $\mathrm{ABC}$ (Atrial Fibrillation Better Care) pathway in clinically complex patients with atrial fibrillation: a post hoc ancillary analysis from the AFFIRM trial. J Am Heart Assoc 2020;9(10):e014932

32 Yang P-S, Sung J-H, Jang E, et al. The effect of integrated care management on dementia in atrial fibrillation. J Clin Med 2020;9 (06):1696

33 Pastori D, Farcomeni A, Pignatelli P, Violi F, Lip GY. ABC (Atrial fibrillation Better Care) pathway and healthcare costs in atrial fibrillation: the ATHERO-AF study. Am J Med 2019;132(07):856-861 